\title{
openheart Quantitative assessment of pericardial delayed hyperenhancement helps identify patients with ongoing recurrences of pericarditis
}

\author{
Arnav Kumar, ${ }^{1}$ Kimi Sato, ${ }^{2}$ Beni Rai Verma, ${ }^{2}$ Chandra Kanth $\mathrm{Ala},{ }^{2}$ Jorge Betancor, ${ }^{2}$ \\ Edlira Yzeiraj, ${ }^{2}$ Lin Lin, ${ }^{2}$ Divyanshu Mohananey, ${ }^{2}$ Salima Qamruddin, ${ }^{2}$ \\ Apostolos Kontzias, ${ }^{3}$ Michael A Bolen, ${ }^{4}$ Massimo M Imazio, ${ }^{5}$ Deborah H Kwon, ${ }^{2}$ \\ Rory Hachamovitch, ${ }^{2}$ Allan L Klein ${ }^{2}$
}

To cite: Kumar A, Sato K, Verma BR, et al. Quantitative assessment of pericardial delayed hyperenhancement helps identify patients with ongoing recurrences of pericarditis. Open Heart 2018;5:e000944. doi:10.1136/ openhrt-2018-000944

$\mathrm{AK}$ and $\mathrm{KS}$ contributed equally.

Received 3 0ctober 2018 Revised 19 0ctober 2018 Accepted 12 November 2018

Check for updates

\section{(c) Author(s) (or their}

employer(s)) 2018. Re-use permitted under CC BY-NC. No commercial re-use. See rights and permissions. Published by BMJ.

For numbered affiliations see end of article.

Correspondence to Dr Allan L Klein; kleina@ccf.org

\section{ABSTRACT}

Objectives Recurrences of pericarditis (RP) are often difficult to diagnose due to lack of clinical signs and symptoms during subsequent episodes. We aimed to investigate the value of quantitative assessment of pericardial delayed hyperenhancement (DHE) in diagnosing ongoing recurrences of pericarditis.

Methods Quantitative DHE was measured in 200 patients with established diagnosis of RP using cardiac MRI. Conventional clinical criteria for diagnosis of pericarditis were $\geq 2$ of the following: chest pain, pericardial rub, ECG changes and new or worsening pericardial effusion. Results A total of 67 (34\%) patients were identified as having ongoing episode of recurrence at the time of DHE measurements. In multivariable analysis, chest pain (OR: $10.9, p<0.001)$ and higher DHE (OR: 1.32, $p<0.001)$ were associated with ongoing recurrence of RP. Addition of DHE to conventional clinical criteria significantly increased the ability to diagnose ongoing recurrence (net reclassification improvement (NRI): 0.80, $p<0.001$; integrated discrimination improvement (IDI): $0.12, p<0.001)$. Among 150 patients with history of RP who presented with chest pain, higher DHE was still independently associated with ongoing recurrence (OR: $1.28, p<0.001)$, showed incremental value over clinical criteria (NRI: $0.76, p<0.001$; IDI: $0.13, p<0.001$ ) and demonstrated a sensitivity of $70 \%$ and specificity of $74 \%$.

Conclusion Among patients with RP, quantitative DHE provided incremental information to diagnose ongoing recurrences over conventional clinical criteria of pericarditis. Quantitative DHE demonstrated acceptable test characteristics to diagnose ongoing recurrence even in RP patients presenting with chest pain.

\section{INTRODUCTION}

Pericarditis is clinically diagnosed when at least two of the four following clinical criteria are met: (1) pericarditic chest pain, (2) presence of pericardial rub, (3) presence of ECG changes (eg, widespread ST-segment elevation and/or recurrent pericarditis (RP) depression) and (4) new or worsening

\section{Key questions}

What is already known about this subject?

- Conventional clinical criteria for diagnosis of pericarditis is met when patients have $\geq 2$ of the following: chest pain, pericardial rub, ECG changes and new or worsening pericardial effusion. However, patients with recurrent pericarditis (RP) present with chest pain even when they are not having an ongoing recurrence of RP and often do not meet the conventional clinical criteria of pericarditis even when they are having another episode of recurrence.

What does this study add?

- Our study indicates that a quantitative assessment of pericardial inflammation using delayed hyperenhancement (DHE) imaging can be used as an objective marker of ongoing pericardial inflammation in patients with established diagnosis of RP. DHE retains good diagnostic value even in patients with RP who present with chest pain. DHE followed improvement in symptoms and serum maker of pericardial inflammation over time.

How might this impact on clinical practice?

- Our data demonstrate that quantitative DHE can help establish the diagnosis of an ongoing recurrences in patients with history of RP. Furthermore, DHE might be a useful marker of pericardial inflammation even in patients with RP presenting with chest pain and help in titration of treatment.

pericardial effusion by echocardiography. ${ }^{1}$ Yet, diagnosing subsequent recurrences of pericarditis in patients with established history of RP is challenging as these patients often have chest pain regardless of their recurrence status. Furthermore, often patients with history of RP who are on multiple anti-inflammatory medications lack enough clinical evidence to meet the clinical criteria of pericarditis. However, accurate decision making 
is critical, as the rate of relapse increases by $50 \%$ after the first recurrence, contributing to the high morbidity of RP. ${ }^{2-4}$ Recent guideline documents have suggested using imaging and laboratory markers of inflammation as additional evidence to diagnose pericarditis. ${ }^{15}$ Indeed, cardiac MRI (CMRI) is increasingly used as a diagnostic tool in a variety of pericardial and myocardial diseases, as it combines excellent anatomic details with tissue characterisation ${ }^{5-7}$ Furthermore, intense pericardial delayed hyperenhancement (DHE) on CMRI represents objective evidence of pericardial inflammation. ${ }^{89}$ Consequently, we aimed to investigate the value of quantitative assessment of pericardial DHE to diagnose an ongoing episode of recurrence in patients with established history RP. Furthermore, we hypothesised that quantitative DHE can add incremental value to conventional clinical criteria for diagnosing an ongoing episode of recurrence of RP and help titrate anti-inflammatory therapy.

\section{METHODS}

The data that support the findings of the study are available from the corresponding author on reasonable request.

\section{Study population and study design}

In a retrospective study, we identified 252 consecutive patients with established history of RP from 1 January 2007 to 28 February 2016, who underwent a CMRI study, had inflammatory markers (ultrasensitive C reactive protein (us-CRP) or Westergren sedimentation rate (WSR)) drawn and echocardiographic assessment done on their first clinic visit at our pericardial centre. Patients were excluded if they had prior history of pericardiectomy or lacked baseline DHE sequences. A pericardial expert (ALK) adjudicated if a patient was having an ongoing episode of recurrence of RP at the time of first CMR based on expert judgement. Patients were only adjudicated as having an ongoing episode of recurrence if they also required an increased dose or additional anti-inflammatory agent to treat an ongoing recurrence per the pericardial expert. Diagnostic accuracy by clinical findings, laboratory markers and imaging variables were compared. Clinical and demographic data were obtained via manual extraction from electronic medical records.

In a subgroup of RP patients with at least one follow-up CMR within 1 year, we also obtained clinical, laboratory and imaging data at the follow-up visit. The study protocol was approved by the Institutional Review Board with a waiver of the requirement for informed consent and patient data were deidentified.

\section{Cardiac magnetic resonance imaging}

All CMR imaging studies were performed using a $1.5 \mathrm{~T}$ MRI scanner (Achieva XR, Philips Medical Systems, Best, The Netherlands) using commercially available software, electrocardiographic triggering and dedicated phasedarray receiver coils. ${ }^{8-11}$
The process of quantitative assessment of pericardial DHE has been described previously. ${ }^{8}$ Briefly, DHE images were obtained in long-axis and short-axis orientations $\approx 10$ min after the intravenous injection of gadolinium-diethylenetriamine penta-acetic acid using a phase-sensitive inversion recovery (PSIR) technique, with inversion time selected for optimal nulling of the myocardium as has been described in previous studies. ${ }^{81213}$ For quantitative DHE analysis, a single observer, blinded to clinical data and outcomes, analysed CMR studies offline using commercially available software (CVI 42; Circle Cardiovascular Imaging, Calgary, Canada). Quantitative analysis of pericardial inflammation was performed on short-axis DHE sequences by manually contouring the pericardium as previously reported. ${ }^{813}$ Normal septal myocardium was then selected as a reference region, and the signal $>6 \mathrm{SD}$ above normal myocardium was quantified. ${ }^{813}$ Qualitative assessment of pericardial DHE was graded as none, mild, moderate or severe by level III expert readers. ${ }^{13}$ The interobserver and intraobserver reproducibility of quantitative assessment of pericardial DHE was excellent and has been reported previously. ${ }^{8}$

\section{Echocardiography}

All patients had a comprehensive echocardiographic examination according to established guidelines. ${ }^{514}$ The presence of pericardial effusion was assessed by two-dimensional echocardiography according to guidelines and semiquantitatively described on the basis of the size of the echo-free space at end-diastole. ${ }^{5}$

\section{Statistical analysis}

Continuous data are expressed as mean \pm SD when normally distributed, or median (IQR) if otherwise. Categorical data are presented as absolute numbers and percentages. The diagnostic value of DHE was compared with clinical, and laboratory markers of pericarditis using logistic regression analysis. In the multivariable model, we included relevant parameters based on previous reports and clinical experience including clinical markers (chest pain, pericardial rub, ECG change and increased effusion), us-CRP, WSR, quantitative DHE, qualitative DHE and pericardial thickness using forward stepwise selection. The optimal receiver operating characteristic (ROC) curve cut-off value for detecting an ongoing recurrence was chosen as the value maximising sensitivity plus specificity. Incremental diagnostic value was defined as the presence of a statistically significant net reclassification improvement (NRI) and integrated discrimination improvement (IDI). Among patients with chest pain, we performed a classification and regression tree analysis to identify the optimal diagnostic modality to differentiate patients presenting with an ongoing recurrence of RP. The results were represented as a binary decision tree.

Longitudinal data analysis of DHE and us-CRP was performed in a subgroup of patients with at least one follow-up CMR using a mixed effect model. To assess the differences in the changes of DHE and us-CRP over time, 


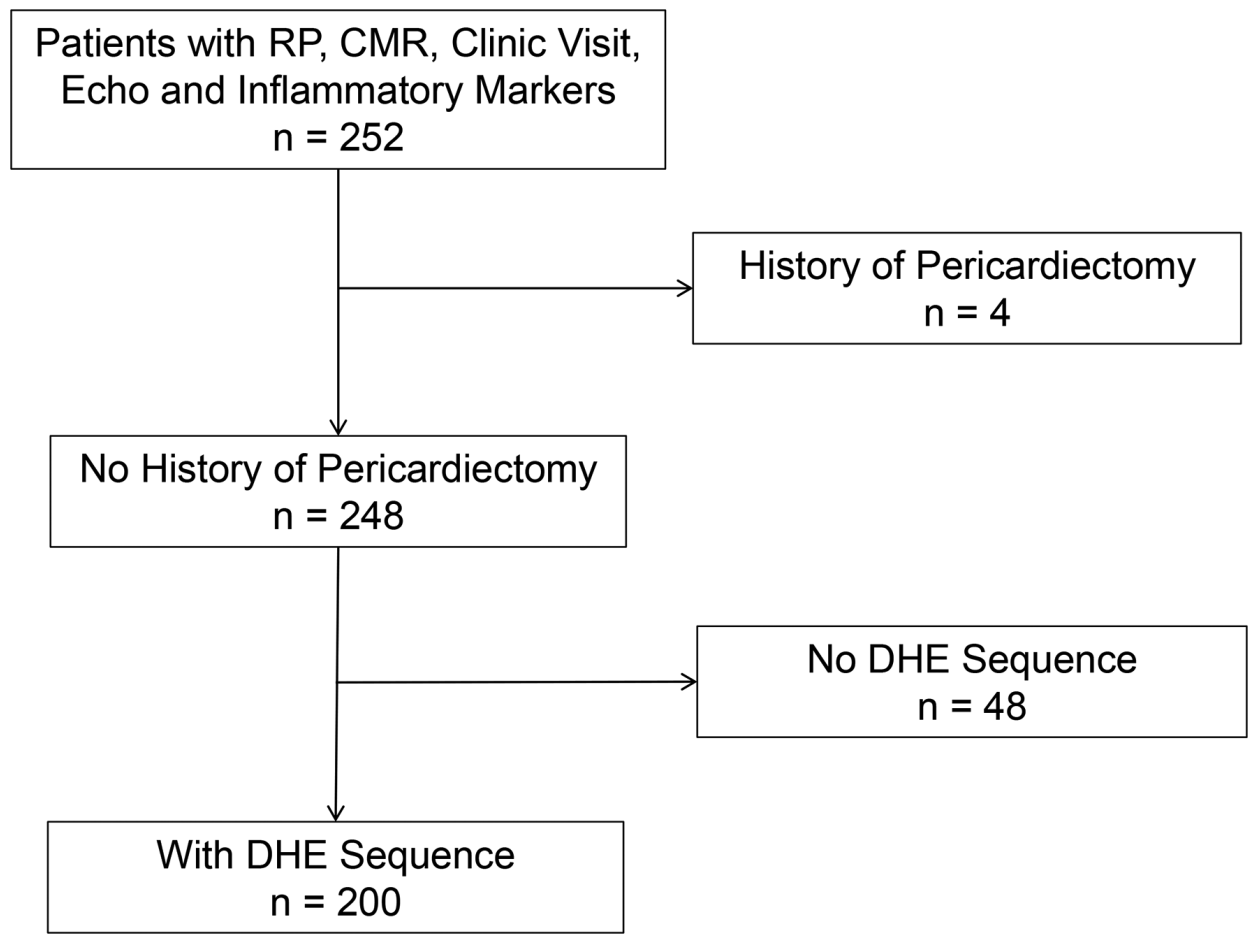

Figure 1 Flow diagram demonstrating derivation of study cohort. CMRI, cardiac MRI; DHE, delayed hyperenhancement; RP, recurrent pericarditis.

a linear mixed effect model was applied with unstructured covariance for random and fixed effects. The slope of regression line obtained by a mixed model are presented along with the corresponding SEs of the actual measurements to show change of parameters over time. All statistical analyses were performed with JMP V.10.0 (SAS Institute, Cary, North Carolina, USA), SPSS 23.0 software and R software V.3.2.2 (R Foundation for Statistical Computing, Vienna, Austria).

\section{RESULTS}

\section{Patient characteristics}

Of the $252 \mathrm{RP}$ patients with CMR assessment, 48 patients were excluded because of absence of baseline DHE sequence or inadequate image quality and four patients due to previous history of pericardiectomy. Finally, 200 patients with established history of RP met the inclusion criteria for our study (figure 1). The median time from initial diagnosis of pericarditis to undergoing initial CMR was 15 (IQR 6-116) months. A total of $150(75 \%)$ patients with RP presented with chest pain at the time of initial CMR and 183 (92\%) were already on anti-inflammatory therapy at the time of initial CMR. Table 1 demonstrates baseline demographics and clinical characteristics of all patients with RP at the time of initial CMR.

At the time of initial CMR, a total of 67 patients were adjudicated as having an ongoing episode of recurrence of RP by the pericardial disease expert of which, 47 (70\%) required addition of a new anti-inflammatory agent and $20(30 \%)$ had their medication dose increased. Of the 67 patients with ongoing recurrence of RP, 63 (95\%)
Table 1 Baseline characteristics of patients with RP during initial CMR

\begin{tabular}{|ll}
\hline & $\mathbf{N}=\mathbf{2 0 0}$ \\
\hline Age, years & $46 \pm 14$ \\
\hline Female gender, $\mathrm{n}(\%)$ & $104(52)$ \\
\hline Chest pain, $\mathrm{n}(\%)$ & $150(75)$ \\
\hline NYHA class (II-IV), $\mathrm{n}(\%)$ & $103(52)$ \\
\hline Duration since initial diagnosis of pericarditis, months & $15(6-116)$ \\
\hline Number of recurrences before initial CMR & $4(2-6)$ \\
\hline Idiopathic aetiology of pericarditis, $\mathrm{n}(\%)$ & $94(47)$ \\
\hline Hypertension, $\mathrm{n}(\%)$ & $37(19)$ \\
\hline Diabetes mellitus, $\mathrm{n}(\%)$ & $16(8)$ \\
\hline Atrial fibrillation, $\mathrm{n}(\%)$ & $22(11)$ \\
\hline Quantitative DHE, cm & \\
\hline Qualitative DHE $>$ none & $50 \pm 36$ \\
\hline Pericardial thickness BB, mm & $145(72.5)$ \\
\hline Anti-inflammatory medications before initial CMR & $2.1 \pm 0.7$ \\
\hline NSAIDs, $\mathrm{n}(\%)$ & $106(53)$ \\
\hline Colchicine, $\mathrm{n}(\%)$ & $142(71)$ \\
\hline Prednisone, $\mathrm{n}(\%)$ & $98(49)$ \\
\hline DMARDs/biologicals, $\mathrm{n}(\%)$ & $18(9)$ \\
\hline
\end{tabular}

Values are mean $\pm \mathrm{SD}$, median $(\mathrm{IQR})$ or $\mathrm{n}(\%)$.

$\mathrm{BB}$, black blood; CMRI, cardiovascular MRI; DHE, delayed hyperenhancement; DMARDs, disease-modifying antirheumatic drugs; NSAIDs, non-steroidal anti-inflammatory drugs; NYHA, New York Heart Association; RP, recurrent pericarditis. 
Table 2 Univariable and multivariable logistic regression model analysis for ongoing recurrence in all patients with recurrent pericarditis $(n=200)$

\begin{tabular}{|c|c|c|c|c|}
\hline & \multicolumn{2}{|l|}{ Univariable } & \multicolumn{2}{|l|}{ Multivariable } \\
\hline & OR (95\% Cl) & $P$ value & OR (95\% Cl) & $P$ value \\
\hline Chest pain & 10.99 (3.79 to 46.69$)$ & $<0.001$ & 10.9 (3.5 to 48.8$)$ & $<0.001$ \\
\hline Pericardial rub & 2.05 (0.47 to 8.92$)$ & 0.33 & - & - \\
\hline ECG change & 4.29 (2.21 to 8.48$)$ & $<0.001$ & - & - \\
\hline Increased effusion on echo & 4.47 (1.83 to 11.71$)$ & 0.001 & - & - \\
\hline us-CRP increase per $10 \mathrm{mg} / \mathrm{L}$ & 1.22 (1.11 to 1.42$)$ & 0.001 & - & - \\
\hline WSR, mm/hour & 1.03 (1.01 to 1.05$)$ & 0.01 & - & - \\
\hline DHE increase per $10 \mathrm{~cm}^{3}$ & 1.32 (1.21 to 1.47$)$ & $<0.001$ & 1.32 (1.19 to 1.48$)$ & $<0.001$ \\
\hline Qualitative DHE & 6.13 (3.14 to 12.33$)$ & $<0.001$ & - & - \\
\hline Pericardial thickness BB, mm & 1.14 (0.76 to 1.72$)$ & 0.51 & - & - \\
\hline At least two positive clinical markers of pericarditis & 5.55 (2.92 to 10.82$)$ & $<0.001$ & - & - \\
\hline
\end{tabular}

BB, black blood; DHE, delayed hyperenhancement; WSR, Westergren sedimentation rate; us-CRP, ultrasensitive $\mathrm{C}$ reactive protein.

patients presented with chest pain, $4(6 \%)$ had pericardial rub, $30(45 \%)$ had ECG changes and $15(22 \%)$ had increased/new effusion on echocardiography. However, $26(45 \%)$ patients adjudicated as having an ongoing recurrence by the expert presented with one or no clinical findings of pericarditis and hence did not meet the conventional clinical criteria of pericarditis. Overall, compared with patients without an ongoing recurrence, patients adjudicated as having a recurrence of RP at the time of initial CMR, demonstrated higher us-CRP, WSR, quantitative DHE and qualitative DHE grade (table 2).

\section{DHE and diagnosing of ongoing recurrences in patients with RP}

In a multivariable logistic regression analysis, presence of chest pain (OR 10.9, 95\% CI 3.5 to $48.8, \mathrm{p}<0.001$ ) and higher quantitative DHE (OR 1.32, 95\% CI 1.19 to $1.48, \mathrm{p}<0.001$ ) were independently associated with an ongoing episode of recurrence of RP (table 2). The optimal cut-off value of DHE for diagnosing an ongoing recurrence of RP was identified as $53 \mathrm{~cm}^{3}$ by ROC curve analysis, showing sensitivity of $70 \%$, specificity of $76 \%$ (positive predictive value (PPV): $59 \%$; negative predictive value (NPV): $83 \%$ ). Adding quantitative DHE to a model meeting the conventional clinical criteria of pericarditis resulted in a significant increase in area under the curve (AUC) ( $\triangle$ AUC: $+0.12, \mathrm{p}<0.001$; figure 2 ) along with significant NRI of 0.80 (95\% CI 0.53 to $1.07, \mathrm{p}<0.001$ ) and IDI of 0.12 (95\% CI 0.07 to $0.18, \mathrm{p}<0.001)$ to diagnose an ongoing episode of recurrence of RP.

\section{Test characteristics of DHE in patients presenting with chest pain}

A total of $150 \mathrm{RP}$ patients presented with chest pain at the time of initial CMR, of which $63(42 \%)$ were adjudicated as having an ongoing recurrence of RP by the pericardial expert. Patients adjudicated as presenting with an ongoing episode of recurrence in this subgroup of patients with RP also demonstrated a significantly higher baseline us-CRP $(41 \pm 66$ vs $10 \pm 38 \mathrm{mg} / \mathrm{L}, \mathrm{p}<0.001)$ and DHE $\left(71 \pm 38\right.$ vs $\left.40 \pm 29 \mathrm{~cm}^{3}, \mathrm{p}<0.001\right)$ when compared with those not having a recurrence. Higher quantitative DHE (OR: 1.28 (95\% CI 1.13 to 1.47 ), $\mathrm{p}<0.001$ ) and presence of at least two clinical markers of pericarditis (OR: 2.53 (95\% CI 1.17 to 5.51), $\mathrm{p}=0.018$ ) were independent predictors of ongoing recurrence in this subgroup of patients (table 3). Even in this subgroup of symptomatic patients with RP, quantitative DHE retained an acceptable test characteristic to diagnose an ongoing recurrence of RP with a sensitivity of $70 \%$ and specificity of $74 \%$ (PPV: 67\%, NPV: 77\%). Similarly, quantitative DHE demonstrated an incremental value over conventional clinical criteria of pericarditis to diagnose an ongoing recurrence of RP with a significant increase in AUC ( $\triangle \mathrm{AUC}$ : +0.11, $\mathrm{p}=0.001$; figure 3$)$, NRI (0.76 (95\% CI 0.46 to 1.06$)$, $\mathrm{p}<0.001)$ and IDI $(0.13$ (95\% CI 0.07 to 0.19$), \mathrm{p}<0.001)$.

\section{CMRI-guided treatment of RP}

A total of 115 patients with RP had follow-up CMRs within 1 year of undergoing the initial CMR. The median time for follow-up CMRI was 147 days (IQR 118-211). At the time of second CMR, the total number of patients complaining of chest pain decreased to 51 (44\%) when compared with $89(78 \%)$ during the initial CMRI $(\mathrm{p}<0.001)$. Longitudinal data analysis revealed that in this subgroup of RP patients with follow-up data, overall DHE significantly improved over time (DHE decrease per 6 months $=-9 \mathrm{~cm}^{3}, \mathrm{p}=0.013$, figure 4). Similarly, overall serum us-CRP levels also improved over time (us-CRP $-16.6 \mathrm{mg} / \mathrm{L}$ at first 6 months, $\mathrm{p}=0.031$ ).

Figure 5A, B shows DHE images of a 47-year-old female patient with RP who presented with chest pain, did not meet conventional clinical criteria of pericarditis and had minimal pericardial DHE (quantitative $\mathrm{DHE}=2 \mathrm{~cm}^{3}$ ) at the time of initial CMR. This patient did not present with an ongoing episode of recurrence at the time of initial CMRI per the pericardial expert and 


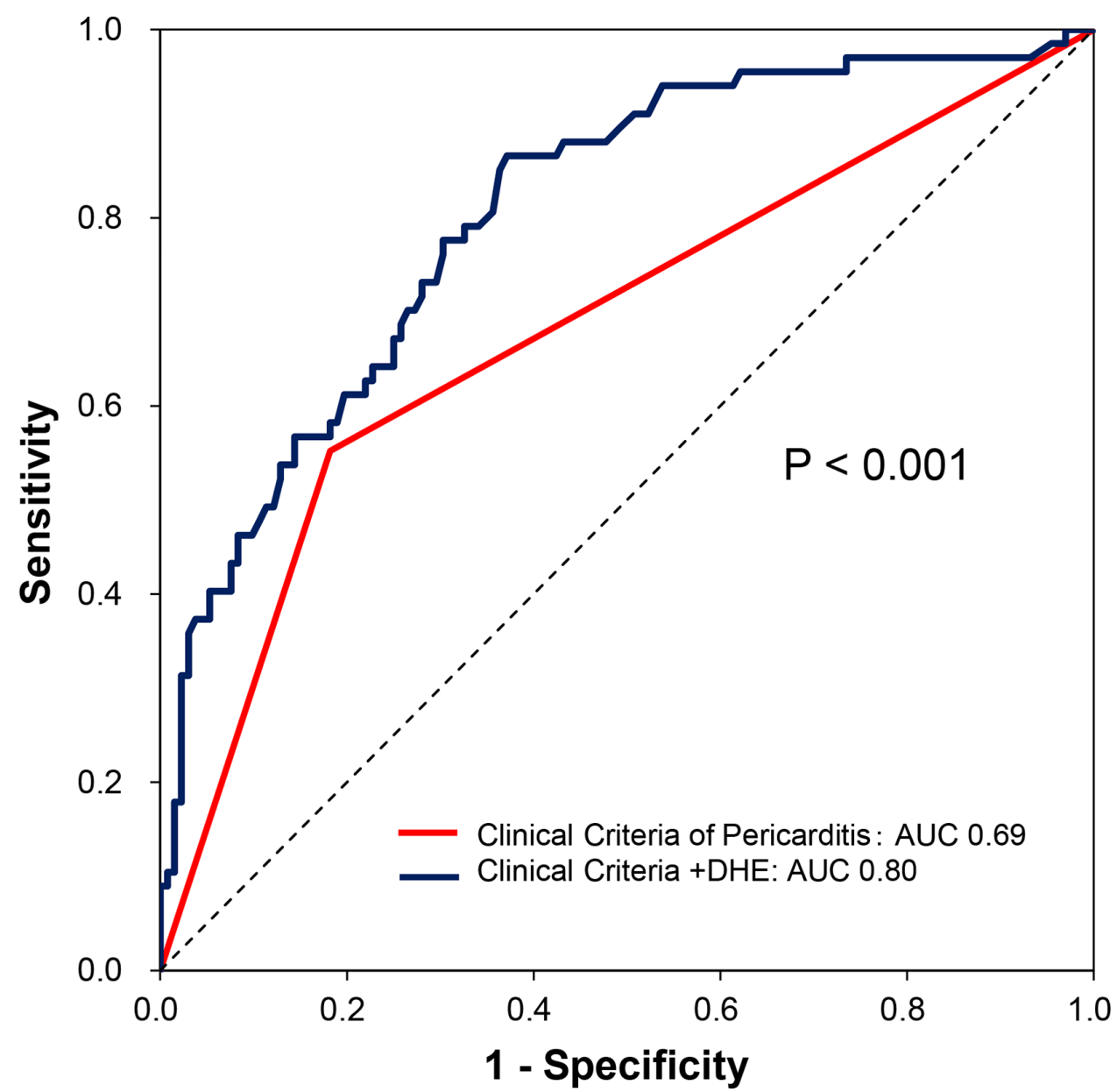

Figure 2 ROC curves showing additive value of DHE in diagnosing ongoing episode of recurrence among all patients with established history of recurrent pericarditis. Red: conventional clinical criteria of pericarditis (AUC 0.69); blue: conventional clinical criteria of pericarditis+DHE (AUC 0.80). AUC, area under the curve; DHE, delayed hyperenhancement; ROC, receiver operating characteristic.

hence did not require further intensification of anti-inflammatory therapy. In contrast, figure $5 \mathrm{C}-\mathrm{F}$ shows DHE images of a 61-year-old female patient with RP who also complained of chest pain and had intense DHE at initial CMRI $\left(\mathrm{DHE}=142 \mathrm{~cm}^{3}\right)$ figure $5 \mathrm{C}$, D. This patient had an ongoing recurrence of RP per the pericardial expert but lacked additional clinical findings of pericarditis. The patient demonstrated an improvement in DHE (quantitative DHE at follow-up $\mathrm{CMR}=34 \mathrm{~cm}^{3}$ ) figure $5 \mathrm{E}, \mathrm{F}$ along

Table 3 Univariable and multivariable logistic regression model analysis for ongoing recurrence in patients with chest pain $(n=150)$

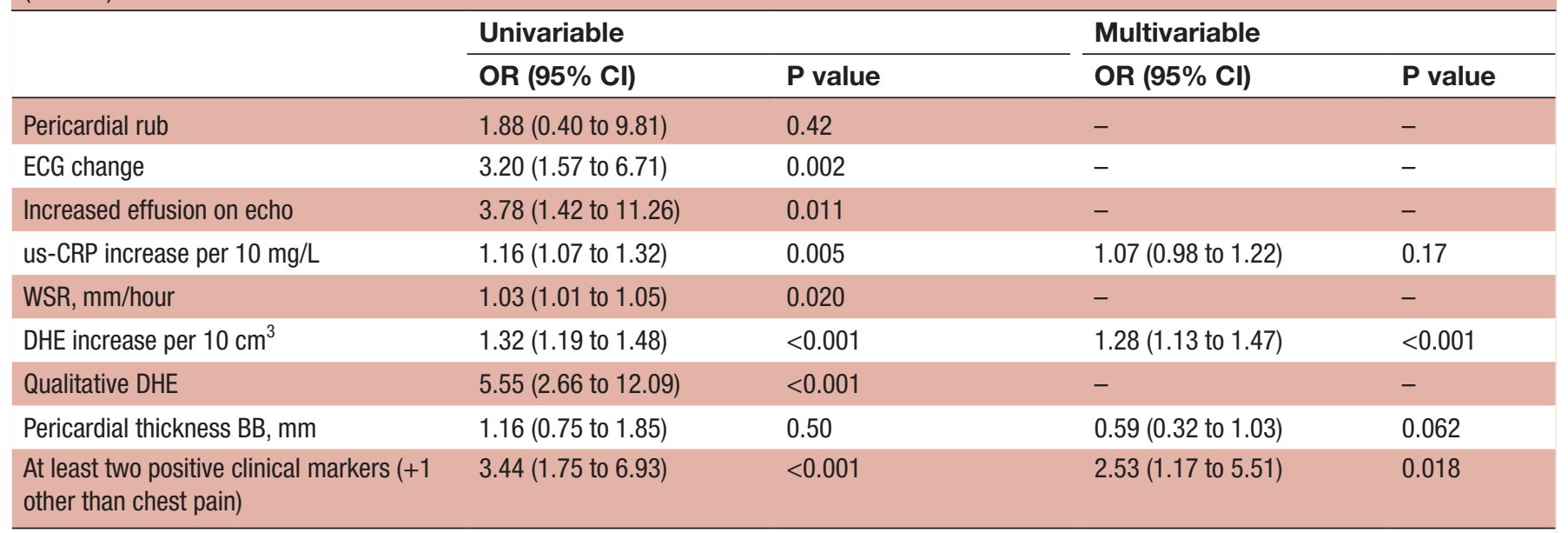

$\mathrm{BB}$, black blood; DHE, delayed hyperenhancement; ROC, receiver operating characteristic; WSR, Westergren sedimentation rate; usCRP, ultrasensitive $\mathrm{C}$ reactive protein. 


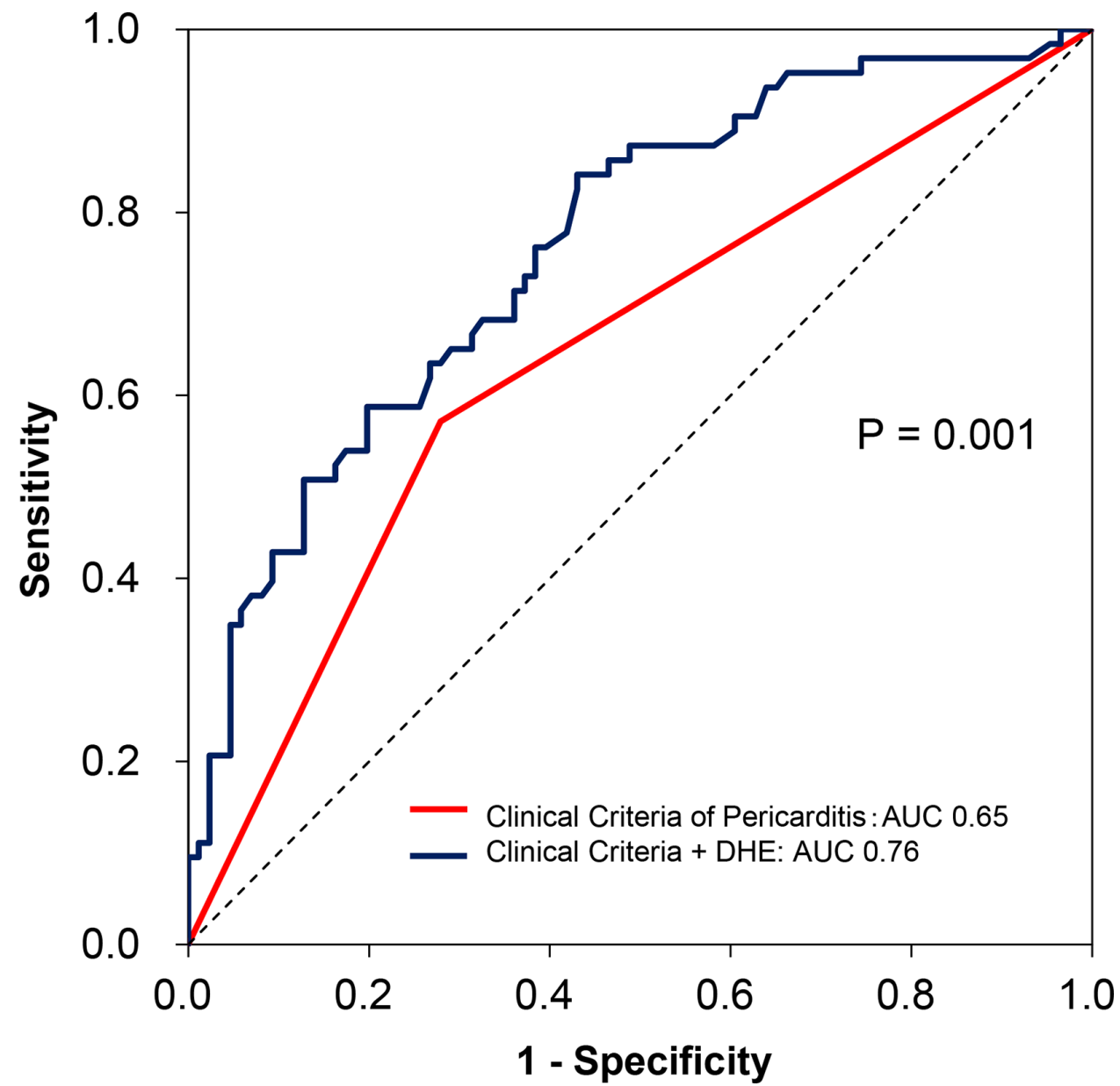

Figure 3 ROC curves showing additive value of DHE in diagnosing ongoing episode of recurrence of recurrent pericarditis in patients who presented with chest pain $(n=150)$. Red: conventional clinical criteria of pericarditis (AUC 0.65); blue: conventional clinical findings+DHE (AUC 0.76). AUC, area under the curve; DHE, delayed hyperenhancement; ROC, receiver operating characteristic.

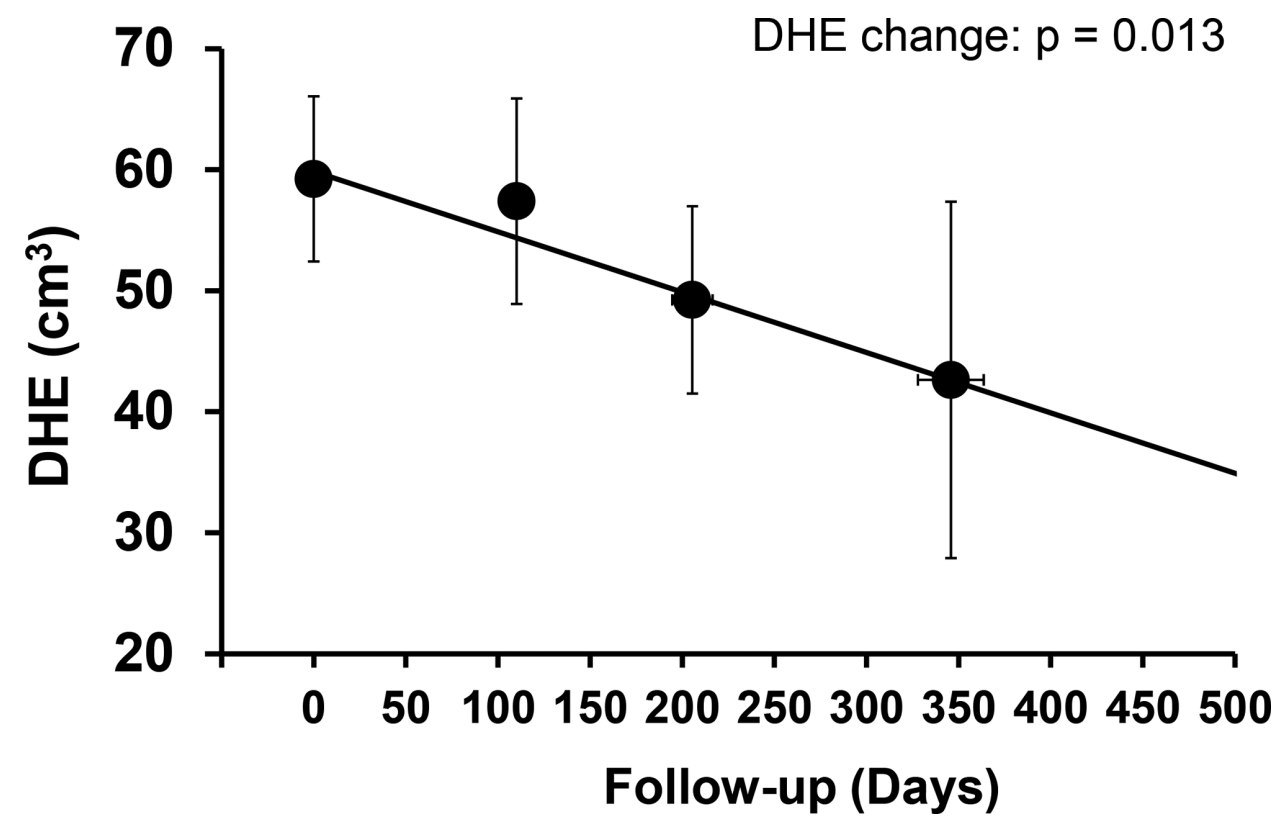

Figure 4 Changes of DHE over time in patients with follow-up CMRI. Markers represent the average of the observed data obtained index CMRI date (time zero) over the intervals of 0-150 days, 151-300 days and $>300$ days. Error bars represent $95 \% \mathrm{Cls}$. The regression line is obtained by the mixed model approach. The P values for change over time are shown. CMRI, cardiovascular MRI; DHE, delayed hyperenhancement. 

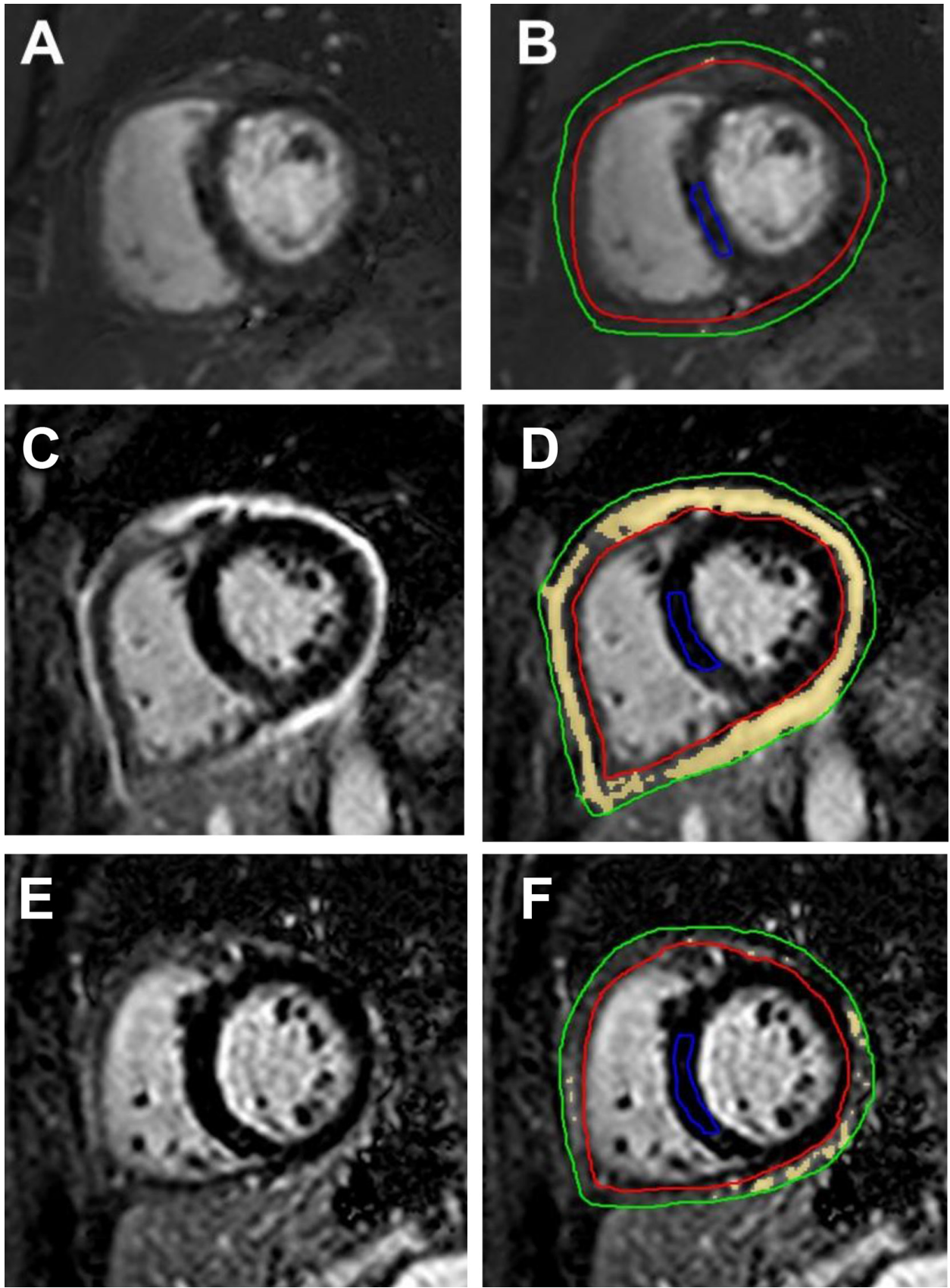

Figure 5 Delayed hyperenhancement (DHE) images from patients with RP. Panels A and B are DHE images from a 47-yearold female patient with RP who had minimal pericardial DHE at presentation. Panels C and D show severe pericardial DHE in a 61-year-old female patient with RP diagnosed as having an ongoing recurrence at presentation, while panels $E$ and $F$ are images from the same patient showing improved DHE post-treatment. Panels $\mathrm{A}, \mathrm{C}$ and $\mathrm{E}$ show images before contouring, and the pericardium is bright from intense DHE in panel C. Postcontouring (B, D and F), quantitative signal $>6 \mathrm{SD}$ above normal myocardium is shown as yellow. On these short-axis images, the pericardium has been outlined between the green and red tracings, and normal septal myocardium has been outlined as a reference region (blue tracing). While DHE images show very low quantitative $\mathrm{DHE}$ (quantitative $\mathrm{DHE}=2 \mathrm{~cm}^{3}$ ) in panel $\mathrm{B}$, panel $\mathrm{D}$ shows high-quantitative $\mathrm{DHE}$ (quantitative $\mathrm{DHE}=142 \mathrm{~cm}^{3}$ ). In comparison with panel D, panel F shows improved DHE (quantitative $\mathrm{DHE}=34 \mathrm{~cm}^{3}$ ) after 200 days of anti-inflammatory therapy. $\mathrm{RP}$, recurrent pericarditis.

with resolution of chest pain at follow-up after 200 days of appropriate medical therapy.

\section{DISCUSSION}

The results of this study demonstrate that in patients with established history of RP: (1) higher quantitative pericardial DHE was independently associated to ongoing recurrence of RP; (2) quantitative pericardial DHE added incremental value in identifying patients presenting with an ongoing episode of recurrence over conventional diagnostic clinical criteria for pericarditis; (3) quantitative DHE retained acceptable discriminatory test characteristics for diagnosing ongoing recurrence of RP among patients presenting with chest pain; and (4) quantitative 
DHE values improved in a subgroup of patients with follow-up CMRI along with improvement in us-CRP levels and symptoms over time.

\section{Diagnostic utility of DH imaging in RP}

$\mathrm{RP}$ is diagnosed when pericarditis recurs after a symptom free period of at least 4 weeks. ${ }^{1}$ However, conventional clinical findings for diagnosing pericarditis are often not observed in subsequent episodes of recurrence of RP leading to inadequate therapy. ${ }^{15} 16$ Not surprisingly, almost half of the patients adjudicated as having an ongoing recurrence by the pericardial expert in our study, did not demonstrate a minimum of two clinical findings of pericarditis at presentation and hence did not meet the conventional clinical criteria to diagnose a recurrence of RP. Previous reports have also documented, that in most recurrences, pericardial chest pain is the only presenting clinical finding. ${ }^{151718}$ Hence, our results were in line with prior reports. In addition, we also demonstrate that adding quantitative DHE to the conventional clinical criteria of pericarditis can improve our ability to diagnose an ongoing episode of recurrence and hence differentiate patients who necessitate intensification of anti-inflammatory medications. Among patients with pericarditis, DHE can inform the presence and severity of pericardial inflammation. ${ }^{19}$ Indeed, patients with RP who have higher DHE at presentation have more active pericarditis and hence demonstrate a higher subsequent recurrence rate. ${ }^{8}$ The results of our study show that DHE imaging might help in diagnosing and treating ongoing recurrences of pericarditis in patients with established history of RP. Interestingly, we found that laboratory markers of inflammation were not independently associated with ongoing recurrences. Most patients in our study were already on anti-inflammatory medications for a considerable period when undergoing the initial CMR. us-CRP is a sensitive marker of inflammation with a short half time, which normalises sooner after initiation of anti-inflammatory medications. Therefore, among patients with RP, who present with recurrences despite being on anti-inflammatory therapy, DHE might be a better marker of disease activity than laboratory markers of inflammation such as us-CRP. ${ }^{8}$

A previous report has demonstrated that higher baseline DHE is associated with a longer clinical course and hence could be used as a prognostic tool in patients with RP. ${ }^{8}$ In the current study, we show for the first time that DHE can be used to identify patients presenting with an ongoing episode of recurrence and titrate anti-inflammatory medications. Furthermore, quantitative assessment of pericardial DHE could be used to provide objective evidence of ongoing pericardial inflammation and add to the diagnostic value of conventional clinical markers of pericarditis in a large cohort of patients with $\mathrm{RP}$ presenting with chest pain. Of note, qualitative DHE reads and laboratory markers of inflammation (us-CRP and WSR) were not found to be independently associated to recurrence of RP in our study.

\section{The paradigm of DHE imaging in pericarditis}

Among patients with pericardial diseases, CMRI can be used to assess haemodynamics, measure pericardial thickness and characterise pericardial oedema and inflammation. ${ }^{5}$ All PSIR DHE images are acquired during diastole when the heart is relatively still and hence the assessment of quantitative DHE is highly reliable and has low observer variability. ${ }^{811}$ Importantly, a normal pericardium is relatively avascular and does not enhance. ${ }^{819}$ However, repeated inflammation of the pericardium as seen in patients with multiple recurrences of pericarditis leads to neovascularisation and uptake of gadolinium-based contrast agents. ${ }^{919}$ Such patients when presenting with recurrent disease show intense DHE suggests the need for intensification of anti-inflammatory therapy.

Conversely, RP patients without significant DHE are more likely to have organised fibrous pericarditis characterised by pericardial fibrosis and calcification. ${ }^{9}$ Of note, patients with constrictive pericarditis who have increased DHE at presentation usually show improvement after a trial of anti-inflammatory therapy. ${ }^{1320}$ However, among patients with RP, symptomatic improvement is often followed by improvement in quantitative DHE levels as evidenced in our study. Whether a DHE-guided approach will lead to overall improvement in outcomes among patients with RP will require further investigation.

\section{Study limitations}

Our study is limited by its retrospective nature. The gold standard for adjudication of ongoing reccurences in our study was based on the clinical judegment of an experienced pericardial expert which could be subjective. The pericardial expert was not blinded to CMRI and echocardiography at patient presentation; however, he had no access to quantitative DHE measurements that were done offline at a later time. To the best of our knowledge, we report the largest cohort of patients with RP who underwent CMR, yet the number of patients is relatively small and comes from a tertiary referral pericardial centre and hence might suffer from selection bias. Furthermore, it is possible that only patients with more advanced disease underwent follow-up CMR, and hence, the overall improvement in symptoms and DHE might be more than that demonstrated in the present study. Indeed, as RP is associated with low mortality rates but has high morbidity, future studies assessing the interval changes of DHE in response to treatment, combined with quality of life matrices are required.

\section{CONCLUSION}

In patients with established diagnosis of RP, quantitative assessment of pericardial inflammation using DHE imaging can help identify patients presenting with an ongoing episode of recurrence of RP. Quantitative DHE assessment was independently associated to ongoing recurrence and added incremental value to conventional clinical criteria of pericarditis. Quantitative DHE 
demonstrated acceptable test characteristics to diagnose ongoing recurrence of RP even in patients presenting with chest pain. Among a subgroup of RP patients with follow-up CMR, a DHE improvement followed improvement in laboratory markers of pericardial inflammation and symptoms.

\section{Author affiliations}

${ }^{1}$ Andreas Gruentzig Cardiovascular Center, Division of Cardiology, Emory University School of Medicine, Atlanta, Georgia, USA

${ }^{2}$ Department of Cardiovascular Imaging, Center for the Diagnosis and Treatment of Pericardial Diseases, Heart and Vascular Institute, Cleveland Clinic, Cleveland, Ohio, USA

${ }^{3}$ Department of Rheumatology, Cleveland Clinic, Cleveland, Ohio, USA

${ }^{4}$ Imaging Institute, Cleveland Clinic, Cleveland, Ohio, USA

${ }^{5}$ University Cardiology, Department of Medical Sciences, AOU Città della Salute $\mathrm{e}$ della Scienza di Torino, University of Torino, Torino, Italy

Contributors AK and KS: conception of the study, data collection, data analysis and writing the manuscript. BRV, CKA, JB, EY and LL: data collection and critical review of the manuscript. DM, SQ, AK, MAB and MMI: critical review of the manuscript. DHK: conception of quantitative DHE methodology, review of data, critical review of the manuscript. $\mathrm{RH}$ : data analysis and critical review of the manuscript. ALK: critical review of the manuscript and responsible for the overall content as guarantor.

Funding The authors have not declared a specific grant for this research from any funding agency in the public, commercial or not-for-profit sectors.

Competing interests None declared.

Patient consent Not required.

Ethics approval Cleveland Clinic Foundation.

Provenance and peer review Not commissioned; externally peer reviewed.

Data sharing statement No additional data are available.

Open access This is an open access article distributed in accordance with the Creative Commons Attribution Non Commercial (CC BY-NC 4.0) license, which permits others to distribute, remix, adapt, build upon this work non-commercially, and license their derivative works on different terms, provided the original work is properly cited, appropriate credit is given, any changes made indicated, and the use is non-commercial. See: http://creativecommons.org/licenses/by-nc/4.0

\section{REFERENCES}

1. Adler Y, Charron P, Imazio M, et al. 2015 ESC Guidelines for the diagnosis and management of pericardial diseases: The Task Force for the Diagnosis and Management of Pericardial Diseases of the European Society of Cardiology (ESC)Endorsed by: The European Association for Cardio-Thoracic Surgery (EACTS). Eur Heart $J$ 2015;36:2921-64.

2. Khandaker MH, Schaff HV, Greason KL, et al. Pericardiectomy vs medical management in patients with relapsing pericarditis. Mayo Clin Proc 2012;87:1062-70.
3. Imazio M, Belli R, Brucato A, et al. Efficacy and safety of colchicine for treatment of multiple recurrences of pericarditis (CORP-2): a multicentre, double-blind, placebo-controlled, randomised trial. Lancet 2014;383:2232-7.

4. Cremer PC, Kumar A, Kontzias A, et al. Complicated Pericarditis: Understanding Risk Factors and Pathophysiology to Inform Imaging and Treatment. J Am Coll Cardiol 2016;68:2311-28.

5. Klein AL, Abbara S, Agler DA, et al. American Society of Echocardiography clinical recommendations for multimodality cardiovascular imaging of patients with pericardial disease: endorsed by the Society for Cardiovascular Magnetic Resonance and Society of Cardiovascular Computed Tomography. J Am Soc Echocardiogr 2013;26:965-1012.

6. Yzeiraj E, Kumar A, Kontzias A, et al. Pericardial enhancement using multimodality imaging in a rare auto-inflammatory disorder. Int $\mathrm{J}$ Cardiol 2016;220:654-5.

7. Bogaert J, Francone M. Pericardial disease: value of CT and MR imaging. Radiology 2013;267:340-56.

8. Kumar A, Sato K, Yzeiraj E, et al. Quantitative Pericardial Delayed Hyperenhancement Informs Clinical Course in Recurrent Pericarditis. JACC Cardiovasc Imaging 2017;10:1337-46.

9. Zurick AO, Bolen MA, Kwon DH, et al. Pericardial delayed hyperenhancement with CMR imaging in patients with constrictive pericarditis undergoing surgical pericardiectomy: a case series with histopathological correlation. JACC Cardiovasc Imaging 2011;4:1180-91.

10. Bolen MA, Rajiah P, Kusunose K, et al. Cardiac MR imaging in constrictive pericarditis: multiparametric assessment in patients with surgically proven constriction. Int J Cardiovasc Imaging 2015;31:859-66.

11. Kramer CM, Barkhausen J, Flamm SD, et al. Standardized cardiovascular magnetic resonance (CMR) protocols 2013 update. $J$ Cardiovasc Magn Reson 2013;15:91.

12. Schulz-Menger J, Bluemke DA, Bremerich J, et al. Standardized image interpretation and post processing in cardiovascular magnetic resonance: Society for Cardiovascular Magnetic Resonance (SCMR) board of trustees task force on standardized post processing. $J$ Cardiovasc Magn Reson 2013;15:35

13. Cremer PC, Tariq MU, Karwa A, et al. Quantitative assessment of pericardial delayed hyperenhancement predicts clinical improvement in patients with constrictive pericarditis treated with antiinflammatory therapy. Circ Cardiovasc Imaging 2015;8.

14. Lang RM, Badano LP, Mor-Avi V, et al. Recommendations for cardiac chamber quantification by echocardiography in adults: an update from the American Society of Echocardiography and the European Association of Cardiovascular Imaging. J Am Soc Echocardiogr 2015;28:1-39.

15. Soler-Soler J, Sagristà-Sauleda J, Permanyer-Miralda G. Relapsing pericarditis. Heart 2004;90:1364-8.

16. Raatikka M, Pelkonen PM, Karjalainen J, et al. Recurrent pericarditis in children and adolescents: report of 15 cases. J Am Coll Cardiol 2003;42:759-64.

17. Imazio M, Gribaudo E, Gaita F. Recurrent Pericarditis. Prog Cardiovasc Dis 2017;59:360-8.

18. Fowler NO, Harbin AD. Recurrent acute pericarditis: follow-up study of 31 patients. J Am Coll Cardiol 1986;7:300-5.

19. Taylor AM, Dymarkowski S, Verbeken EK, et al. Detection of pericardial inflammation with late-enhancement cardiac magnetic resonance imaging: initial results. Eur Radiol 2006;16:569-74.

20. Feng D, Glockner J, Kim K, et al. Cardiac magnetic resonance imaging pericardial late gadolinium enhancement and elevated inflammatory markers can predict the reversibility of constrictive pericarditis after antiinflammatory medical therapy: a pilot study. Circulation 2011;124:1830-7. 